\title{
Revista Instantáneas de luz $i$ sombra en la historia de la crítica literaria chilena*
}

\author{
Magazine Instantáneas de luz i sombra in the history \\ of the Chilean literary criticism
}

JAIME GALGANI $^{* *}$
Universidad Católica Silva Henríquez. Santiago, Chile $_{\text {jgalganim@ucsh.cl }}$

\section{RESUMEN}

La historia de la crítica literaria en Chile a menudo comienza con el nombre de Andrés Bello como primer exponente. En algunos casos, hay ciertas búsquedas de testimonios críticos en la Literatura Colonial. En otros, se hace una distinción entre la crítica decimonónica (considerada como "comentario literario") y la crítica profesional del siglo XX, que comenzaría con Omer Emeth hacia 1906. Este artículo plantea que, entre el siglo XX y Omer Emeth, existe un proceso de transición en el que juega un papel clave la existencia de la revista artístico-literaria Instantáneas de luz i sombra. En dicho semanario, se observa un ejercicio crítico que va desde los más elementales comentarios hasta la madurez de una actividad crítica escritural que da signos de avanzada profesionalización.

Palabras claves: Campo literario, crítica literaria, legitimación, delegación, capital específico, revistas literarias.

* Este artículo forma parte de la investigación realizada por el profesor Jaime Galgani en el proyecto que preside: iniciación FONDECyT No11090054, "Literatura chilena y proyecto cultural en revistas de inicio del siglo XX (1900-1910)", y está escrito sobre la base de una ponencia presentada en el "Congreso Internacional Ciudad y los Imaginarios en las Literaturas Latinoamericanas" (noviembre de 2009).

${ }^{* *}$ Doctor en literatura, académico Departamento de Humanidades Universidad Católica Silva Henríquez, jgalganim@ucsh.cl 


\section{ABSTRACT}

The history of the Chilean literary criticism often begins with the name of Andres Bello as the first prominent figure. In some cases, there are searches of critical testimonies in the colonial literature. In other cases, a distinction is made between the decimononical critique (known as "literary commentary") and the professional criticism of the $20^{\text {th }}$ century, which started with Omer Emeth towards 1906. This article states that, between the $20^{\text {th }}$ and Omer Emeth, there exists a transition process within which the emergence of the literary and artistic magazine Instantáneas de luz $i$ sombra plays a crucial role. In that weekly paper, a critical exercise is observed, this goes from the most basic commentaries towards the maturity of written critical practice that shows signs of advanced professionalization.

Keywords: Literary camp, literary criticism, legitimation, delegation, specific capital, literary magazines.

Recibido: 02/08/2010.

Aceptado: 09/02/2011.

\section{INTRODUCCIÓN}

工 a habido distintas posturas frente a la crítica literaria chilena. Algu1 nos, como Maximino Fernández Fraile, se remontan a la misma época colonial, donde, reconociendo que no había crítica literaria profesional, sí había, en ciertos textos, una reflexión por parte del autor de su propia escritura; así, en efecto, sucede con Ercilla, Ovalle, y Pineda y Bascuñán. Otros, como Raúl Silva Castro, consideran que la actividad crítica en Chile comienza con Andrés Bello, iniciándose con él un proceso que habría de tener su maduración con Omer Emeth y se afianzaría durante el siglo XX. John Dyson, por su parte, presenta la historia de la crítica literaria chilena dividida en seis perspectivas metodológicas, llamadas por él "linajes". Gonzalo Catalán, con una tercera visión, más ligada a la teoría de campo de Pierre Bourdieu, demuestra que el verdadero primer crítico profesional en Chile fue Omer Emeth; se apoya para ello en palabras de Alone y llega a establecer que lo que había antes de Omer Emeth (es decir, antes de 1906, aproximadamente) era solamente una suerte de comentario literario.

Revisando esta trayectoria, como parte de una investigación más general, este artículo se plantea, como objetivo, insertar en la historia de la crítica literaria chilena un momento considerado como fundante y que corresponde 
a los dos primeros años del siglo XX, lapso en que, con corta pero fructífera duración, fue publicada la revista Instantáneas de luz $i$ sombra (junto a otras que la antecedieron o le hacían la competencia). Pues bien, este semanario artístico literario, que agrupó en torno a sí nacientes y relevantes exponentes de la historia de las letras chilenas, constituye un venero de desconocidos documentos que pueden dar luz sobre varios aspectos relacionados con el campo literario y su entonces incipiente pero acelerado proceso de autonomización.

Con respecto al objeto analizado, la hipótesis de trabajo de la investigación afirma que, en Instantáneas de luz i sombra, se desarrolla un proceso tal de evolución de la crítica literaria que se constituye en una verdadera transición entre el comentario literario propio del siglo XIX y la crítica especializada del siglo XX, que al interior de la revista se dan muestras de evolución en la forma de concebir la crítica literaria, así como de una conciencia metacrítica explícita, y que, dentro de sus exponentes, el antecedente más maduro de la crítica de inicios de siglo es Augusto Thomson, probablemente más aventajado incluso que Omer Emeth, hasta ahora indiscutido primer gran crítico nacional.

El artículo se propone demostrar las primeras afirmaciones de esta hipótesis, dejando la discusión sobre la superioridad de Thomson sobre Omer Emeth para otro momento y adverbiándola, por eso y por ahora, con un "probablemente" provisional.

La metodología de análisis, de carácter descriptivo-interpretativo, revisa algunas de las historias de la crítica literaria chilena para sumarizar una suerte de estado del arte que comienza con los aportes de Dyson, sigue con los de Silva Castro y concluye con los de Catalán. Posteriormente, siguiendo el mismo camino, se intenta ofrecer elementos de investigaciones más recientes (de Marina Alvarado (su tesis doctoral) y las mías propias) para instalar aquí la cuestión central del artículo, es decir, posicionar el lugar relevante y poco conocido de la revista escogida y de sus actores principales (Thomson, entre ellos).

\section{ANTECEDENTES EN LA HISTORIA DE LA CRÍTICA LITERARIA CHILENA}

Según John P. Dyson, es posible clasificar en seis linajes la crítica literaria chilena, de acuerdo a la pluralidad de enfoques que asume: gramatical, humanístico, histórico, sociológico, impresionista y estético. El linaje gramatical habría comenzado a ser desarrollado por Miguel de Olivares (1713- 
1793), puesto que, en el prefacio de su Historia militar, civil y sagrada del Reino de Chile, nos muestra esa incipiente crítica cuando nos dice que

la piedra de escándalo es el estilo, en que todos querían votar y aún dar preceptos, porque si usa el autor del mediano, lo tienen por bajeza; si del alto, por afectación; si es ceñido, le tachan de falta de amenidad; si es vario, lo culpan de confusión; si deja por decir algo, lo tienen por negligencia; si lo dice todo, por prolijidad (Citado por Dyson, 1965: 25);

de este modo, fijando sus apreciaciones en el estilo, este linaje se orienta principalmente a la factura formal del texto y ya en la época colonial encontraba exponentes en las letras chilenas. El linaje humanístico, en cambio, responde a la apreciación del humanismo como "manifestación renacentista de un nuevo interés en el hombre individual como eje del mundo" (32), considerando, como línea crítica, que "la literatura es esencialmente una parte de la totalidad intelectual y cultural de la humanidad [siendo] una profundización del linaje gramatical, puesto que lleva al terreno filosófico el conjunto de reglas mecánicas de la gramática" (32); el primer exponente de este trabajo crítico en Chile es Andrés Bello (1781-1865). En tercer lugar, con respecto al linaje histórico,

[o] que caracteriza e distingue esta familia é o fato de seus membros encararem a literatura não tanto como fenómeno esencialmente estético, desligado, por consequência, em certa medida, do tempo, mas ao contrario, como un problema de história, que ao tempo deve o seu caráter, e nêle encontra a sua explicação (Wilson Martins, citado por Dyson, 1965: 43).

El fundador de este linaje en Chile sería Ramón Belisario Briseño (18171910), cuyo aporte a la crítica fue el de haber sido el primero en establecer esta familia espiritual con su Estadistica bibliográfica de la literatura chilena (1862-1879) y sus Bocetos literarios (1882). Otros exponentes de la que, según Dyson, es la más nutrida línea de crítica literaria, son Miguel Luis y Gregorio Víctor Amunátegui, Diego Barros Arana y José Toribio Medina. Siguiendo todavía a Dyson, el cuarto linaje, i.e., el sociológico, que considera la literatura como "una manifestación del fenómeno social, o sea, una creación de grupo en la medida en que la colectividad influye sobre el escritor" (58) -el arte como procedimiento sociológico y colectivo- tendría en Chile, como primeros exponentes, a José Victorino Lastarria (1817-1888) quien, en su discurso inaugural de la Sociedad Literaria (1842), plantea: 
Se dice que la literatura es expresión de la sociedad, porque en efecto es el resorte que revela de una manera más explícita las necesidades morales e intelectuales de los pueblos, es el cuadro en que están consignadas las ideas y pasiones, los gustos y opiniones, la religión y las preocupaciones de toda una generación... (citado por Dyson, 1965: 60).

El linaje impresionista, en quinto lugar, que corresponde a la crítica homónima, se asocia al subjetivismo crítico y, en cuanto tal, tiene como objetivo comunicar "las vibraciones de su propia psique [la del crítico] en contacto con una obra determinada" (94). A este linaje corresponde el trabajo de Miguel Luis Rocuant (1877-1948), quien, al hablar de crítica moderna, planteaba: "Hoy ha evolucionado [la crítica]: de analítica, se ha convertido en sintética, en impresionista, y de impresionista, en indiferente [al casticismo y a la temática solamente nacional]" (citado por Dyson, 1965: 94). El último linaje, el estético, es, a juicio de Dyson, el que corresponde al [profesional] crítico "que parece acercarse más al ideal de la crítica literaria, porque sabe que antes que todo un arte estético demanda una crítica de naturaleza idéntica", la cual concibe que "el fenómeno literario es un hecho real que depende solamente de su propia perfección; encuentra su razón de ser y su logro en sí y no en algo extrínseco a él, ni mucho menos al arte en general" (94); primeros exponentes de esta línea son Julio Bañados Espinosa (18581899) y Luis Covarrubias (1865-1916).

Raúl Silva Castro, en el "Estudio preliminar" contenido en su Literatura Critica de Chile (1969), establece que el primero en "aclimatar", en Chile, el oficio asociado a la crítica literaria fue Andrés Bello, quien, en las páginas de El Araucano, no comentó principalmente obras de autores chilenos, dedicándose especialmente a piezas teatrales de autores extranjeros. Según Silva Castro, sería con José Victorino Lastarria (1817-1888) con quien comienza la crítica de autores chilenos, práctica que continuaría con Manuel Blanco Cuartín (1822-1890), "estudioso del teatro de su tiempo y de la poesía española” (12). A ellos se sumaría el trabajo de los hermanos Amunátegui, Miguel Luis (1828-88) y Gregorio Víctor (1830-99) y, posteriormente, Joaquín Blest Gana (1831-1880), Adolfo Valderrama (1834-1902), Zorobabel Rodríguez (1839-1901), Eduardo de la Barra (1839-1900), José Toribio Medina (1852-1931) y otros más, hasta que aparecen las figuras más destacadas de Pedro Nolasco Cruz (1857-1939) y Omer Emeth (Emilio Vaïse, 1860-1935). Silva Castro, sin ofrecer una clasificación minuciosa como la de Dyson, realiza una semblanza de cada uno de los críticos que elenca en su presentación. 
Más cercano a nuestros días, Maximino Fernández Fraile (2003), reconociendo que en los llamados siglos coloniales (XVI, XVII y XVIII) "no hubo crítica literaria" en Chile y que "apenas si algunos de los escritores de aquellos tiempos tuvieron preocupaciones respecto de su propia obra” (49), coincide con la ya clásica afirmación de que con Andrés Bello se asentó la crítica literaria en Chile. Pues bien, tomando tres ejemplos de estos autores coloniales que problematizan su ejercicio literario, ve en ellos una especie de semillas críticas que considera valiosas de rescatar. El primero de ellos es Alonso de Ercilla, quien comienza su obra La Araucana con los versos
No las damas, amor, no gentilezas
de caballeros canto enamorados,
mas el valor, los hechos, las proezas
de aquellos españoles esforzados...

que indican, según Fernández Fraile, una disposición intencional del autor sobre su obra y, en consecuencia, una postura reflexiva ante la misma (2003: 49-50). Un segundo ejemplo es propuesto en Alonso de Ovalle (siglo XVII) quien, "en su Histórica relación del Reino de Chile y de las misiones y ministerios que ejecuta en él la Compañía de Jesús, se preocupó especialmente por el empleo del lenguaje, denotando un dominio absoluto de él” (50). Del mismo modo, comenta a Francisco Núñez de Pineda y Bascuñán, también del siglo XVII, y a su obra Cautiverio feliz, discutiendo sobre ella la cuestión relacionada con el género literario al que pertenece, aspecto no siempre claro en todas las obras y que constituye, en no pocos casos, una pregunta a responder por parte de la crítica literaria.

Fernández Fraile, abandonando el período colonial, afirma que tampoco hubo crítica literaria durante la Guerra de la Independencia y en el período inmediatamente posterior, a pesar de que en los periódicos que comenzaban a editarse (La Aurora de Chile, El Mercurio Chileno, La Clave, El Mercurio de Valparaiso, El Araucano) hubiera algunos comentarios a ciertos poemas o a libros extranjeros, comentarios que a menudo tenían un carácter más ideológico, acordando con las metas presentes en el momento y consistentes con la urgencia de construir la nación. Posteriormente, comienzan a reaparecer los mismos nombres citados por Dyson y Silva Castro, así como lo harán en su momento otros estudiosos.

En síntesis, se podría postular que, al menos hasta iniciada la segunda mitad del siglo XX, hay una suerte de "historia oficial" de la crítica literaria 
en Chile que, sumando o restando, plantea que hay una especie de preparación del terreno con las letras coloniales y el periodismo de la Guerra de la Independencia y décadas posteriores, una primera tentativa crítica representada por el trabajo de Andrés Bello, continuada por José Victorino Lastarria, afianzada por personalidades como los hermanos Amunátegui, Zorobabel Rodríguez, Toribio Medina, y madurada por la aparición de Emilio Vaïse y Pedro Nolasco Cruz, a quienes seguirían, posteriormente, los grandes críticos del siglo XX (Ricardo Latcham, Hernán Díaz Arrieta, Raúl Silva Castro y otros). Todos ellos, siguiendo el modelo de Dyson, se adscribirían a diferentes escuelas de orientación crítica (que él llama linajes), acogiendo perspectivas que pueden ser gramaticales, humanísticas, históricas, sociológicas, impresionistas o estéticas. He aquí la síntesis de la historia de la crítica chilena hasta los años sesenta del siglo XX.

\section{UNA SEGUNDA VISIÓN: OMER EMETH EL PRIMER CRÍTICO PROFESIONAL}

Gonzalo Catalán, en su estudio titulado "Antecedentes sobre la transformación del campo literario en Chile entre 1890 y 1920" (1985), sin desconocer la actividad crítica de los nombres mencionados anteriormente y que se sitúan antes de la la época que analiza, sí postula una inflexión significativa a partir de Omer Emeth, pseudónimo del sacerdote francés Emilio Vaïse, que, aunque no ignorado por ninguno de los historiadores de la crítica literaria chilena, aún no había sido reconocido como el oficiante de un nuevo sistema crítico, es decir, sujeto profesional, voz pretendidamente imparcial y actor de un campo literario que experimentaba su proceso de autonomización en el período en que comienza a publicar.

Antes de Omer Emeth -señala Alone- los críticos eran, generalmente, los autores de novelas, poesías, cuentos o historias que, según la ocasión intercambiaban elogios o censuras de un modo esporádico. No había en los periódicos una sección estable que atrajera a los lectores deseosos de información, faltaba esa espina dorsal que presta coherencia al movimiento de los libros y los obliga a considerarlos incluso a quienes no acostumbran a leer. Omer Emeth creó ese personaje, ingrato para unos, útil para la mayoría, aunque no todos lo reconozcan, llamado el crítico... (Alone, citado por Catalán, 1985: 150). 
En efecto, Omer Emeth, quien aparece en la escena literaria hacia 1906 y publica sus críticas especialmente en El Mercurio, se manifiesta como el crítico moderno, cuyo modelo corresponde a las exigencias de un perfil en transformación consistente en "consolidar e imprimir un carácter decididamente institucional a esa función de intermediación entre el consumo y la producción literaria que constituye lo sustancial de la actividad crítica" (Catalán, 1985: 151). Este carácter institucional corresponde a un mercado literario que se ha hecho más masivo, en donde se manifiestan las necesidades de mediación entre el escritor y el lector, y que se formulan bajo los conceptos de delegación y legitimación. La delegación sucede naturalmente al proceso de autonomización del campo literario y supone la presencia de un nuevo oficio que ha de movilizar las regulaciones normales del mercado por la vía del oficio crítico. Como dice Catalán:

Pero no cabe duda que, como ya lo hemos anunciado, el escenario privilegiado de esta delegación -ahí donde ella propiamente se formaliza- corresponderá a los dominios de la crítica literaria, más aún cuanto que la propia crítica deberá su perfil moderno a ese proceso de delegación y a las nuevas condiciones que supone (1985: 149).

Por otro lado, Omer Emeth responderá a una característica fundamental del período que no es otra cosa que la profesionalización del oficio. Es decir, si Augusto D'Halmar se constituye en el primer escritor profesional, y Agustín Edwards Mac Clure en el empresario editorial por excelencia, así Omer Emeth, a diferencias de los críticos del siglo XX, en quienes el trabajo crítico no siempre constituía la centralidad de su contribución cultural, puede afirmarse que es, con toda propiedad, un profesional de la crítica literaria, y en esto comparte con los demás actores del campo cultural un proceso tan propio de la modernidad como es la especialización.

La historia de la crítica literaria chilena contará durante el siglo XX con destacados nombres, los cuales marcarán los parámetros de seguimiento y de juicio de la producción literaria. El crítico, como censor dotado de un cierto privilegio y de una autoridad concedida a su conocimiento de la materia, sufrirá alternativamente de la admiración y el odio de parte de los diversos agentes del campo literario, pero siempre contará con la seguridad de su imprescindibilidad cultural. Junto a Omer Emeth, destacarán los nombres de Pedro Nolasco Cruz y, posteriormente, de Alone y de Ignacio Valente. Desde las últimas décadas del siglo, hasta nuestros días, se diversificará en nombres y posturas. 
En conclusión, siguiendo a Alone y a Catalán, cabe decir que se establecen dos épocas históricas claras: la crítica de viejo cuño, dominada por los sesgos propios de clase vigentes en el siglo XIX y teniendo en cuenta los proyectos nacionales en pugna, una crítica que se ha acordado denominar como "comentario literario", y la crítica moderna, más profesional, independiente, orientada preferentemente a la valoración estética de la obra literaria.

\section{INSTANTÁNEAS DE LUZ I SOMBRA Y SU ROL EN LA FORMACIÓN DE LA CRÍTICA PROFESIONAL}

Pareciera que todo queda dicho con el reconocimiento del lugar que ocupa Omer Emeth en el proceso. Sin embargo, los tratados escritos sobre el tema (como los revisados anteriormente) adolecen, en términos generales, de una grave falencia, la cual consiste en obviar las etapas que generan la aparición de los grandes exponentes. De hecho, pocos artículos advierten la importancia que tuvieron algunas revistas en la conformación de la profesionalización de la crítica literaria en Chile, y ninguno se adentra a especificar procedimientos, personajes y ejemplos. Este es el caso de la publicación Instantáneas de luz $i$ sombra (1900-1901), sobre la cual este artículo quiere hipotetizar que tuvo un rol clave en la transición desde "el comentario literario", que era el modelo crítico correspondiente al siglo XIX, y la crítica profesional que se establece con Omer Emeth.

Alfredo Melossi, hombre culto y emprendedor, convirtió, en marzo de 1900, la hoja gratis "El Turista", con que promocionaba su hotel, en la revista Luz i sombra. Simultáneamente, un animado grupo de escritores e intelectuales daba vida a la revista Instantáneas. En ese mismo año, Luz i sombra se fusionó con Instantáneas, creando Instantáneas de luz i sombra. Paralelamente, y por más tiempo, se publicó la revista Pluma y lápiz, dirigida por Marcial Cabrera, con la cual se estableció una relativa competencia. Estas revistas cumplieron un rol decisivo en la aparición de varios escritores: Augusto D'Halmar, Guillermo Labarca Huberston, Antonio Orrego Barros, Ignacio Pérez Kallens, Carlos Pezoa Véliz, Carlos Mondaca, Ernesto Guzmán, Antonio Bórquez Solar. Algunos de ellos gozaron de una fama que sigue hasta nuestros días; otros han sido olvidados, pero todos contribuyeron a establecer las bases de una nueva configuración del campo literario en Chile.

Las manifestaciones que se acercan a la "crítica literaria", por parte de la revista Instantáneas de luz $i$ sombra, están dadas por el hecho de disponer de 
un apartado y unos responsables destinados a realizar observaciones sobre las participaciones (cuentos, poemas) que llegaban a la revista y que aspiraban a ser publicadas, y por la función crítica mediadora que establecían entre escritores y público lector. Evidentemente, a dicho oficio crítico le falta un componente principal consistente en la perspectiva teórica necesaria para discriminar, puesto que, en general, los comentarios sobre los textos llegados a la redacción no van acompañados más que de un rechazo o una aceptación de los mismos. Esto confirma que se está frente a un período de transición donde se verifica la presencia de un "actor crítico", pero aún no dotado de las características propias de la profesionalización del oficio.

Ahora bien, dentro de los más destacados representantes de este género en ciernes, aparecen los nombres de Federico Matas (que se hace llamar en distintos momentos como Teloncillo, Buenafé, Lladre), Ruy Blas, Víctor Tarugo, Daniel Eyssette, Juan Francisco González, Ignacio Pérez Kallens, Augusto G. Thomson (D’Halmar) y Tío Grulo, curioso pseudónimo de un personaje que nunca se identifica por su nombre real.

Tío Grulo está encargado de una sección llamada "Correspondencia particular", la que comienza a ser publicada en el número trece de la revista Instantáneas, desapareciendo en el número veintiuno. Cuando se produce la fusión de los semanarios, esta sección permanece. Se caracteriza por sufrir variadas modificaciones y no aparecer de manera continua. A lo largo del tiempo, el mismo Tío Grulo se ve en la necesidad de modificar la percepción de su propio trabajo:

Mi amable director ha tenido la cortesía de ascender mi jerarquía de simple llenador de preguntas y respuestas, al de sus redactores, encomendándome el cumplimiento de esta sección, que árida en su comienzo, ha logrado interesar al benévolo lector, llegando algunos a solicitar la publicación constante de ella. Tal petición no será tan fácil de satisfacer semanalmente, pues puede no haber asunto para ello, no obstante que hasta aquí hemos dejado de mano muchas sandeces que por lo insulsas no se prestaban ni siquiera a bromas, que con la mejor intención solemos hacer á algún colaborador que ha equivocado su vocación. Hoy por hoy tenemos nuestra cartera repleta, y por desgracia en su mayor parte poesías, lo que hace pensar, con perdón de nuestros lectores, si no se habrá desarrollado otra peste tan temible como la alfombrilla (ILS 30, 13).

Esta cita da cuenta de que llegaban a la redacción numerosas colaboraciones, lo que testimonia el crecimiento de la producción literaria y el inte- 
rés por publicar y por aparecer en algún espacio de legitimación. Al mismo tiempo, se advierte la predominancia de la poesía por sobre la narración.

Las opiniones de Tío Grulo (que durante la duración de la revista cambia de nombres) son del más variado tipo. Domina en ellas el juicio exento de razonamientos y argumentos. También, en algunos casos, aparece el humor sarcástico:

Sr. E. Diez de M. - La Paz - Por A varios solicitantes. - Muy grato nos sería alentar á la juventud que se inicia en el campo de las letras, pero si se nos permite esta apreciación creemos que no es Instantáneas centro de ensayo, y que bastante hacemos esperar á quienes tienen derecho á un sitio en nuestras páginas para complacer á los novicios que aun nos piden corrijamos sus trabajos (ILS 40, 14).

Señor K. K. O. - Su composición $A$ un ciprés es demasiado fúnebre, y además de fúnebre, mala $(I 13,12)$.

Art. - Señor: ¿Por qué hace V. versos, cuando tiene usted excelentes condiciones para estar en el Manicomio con camisa de fuerza? No se ofenda V.; ¡Es sólo una pregunta! $(I 15,15,14)$.

Zoñum. - No sea un payaso $(I 19,13)$.

D. Mente. - Hace V. honor á su nombre; disculpe la claridad $(I 21,14)$.

Sr. Cáscaras, Talca. - Eso iba á decir yo ¡Cáscaras! ¡Qué malo es esto! (ILS 26, 13).

Sr. F. A. F. - No llame usted á Rosenda en verso, porque si ella se entera no viene, créame usted (ILS 30, 13).

Sr. Galvez-ton. - ¿Con que me autoriza usted para suprimir una estrofa?, ¿eh? Bueno, gracias, lo suprimiremos todo y así queda usted mejor con Natalia (ILS 26, 13).

El tono de las respuestas varía en algunos momentos, indicando criterios que iluminan la estética que lo anima a juzgar un poema. Así, por ejemplo:

Dice La Bruyére, y ustedes los poetas deberían saberlo, acatarlo y no escribir tonterías hasta que no estén medianamente preparados. En ciertas cosas la medianía es soportable: tales son la poesía, la música, la pintura, el discurso público.

No hay suplicio como de oír declamar pomposamente un discurso amanerado y frío, ó el de escuchar la lectura de versos malos, hecha con todo el énfasis de un mal poeta $(I L S 57,13)$. 
Las observaciones hechas indican un rechazo hacia el amaneramiento, el histrionismo, la oratoria artificial y sobreactuada, el afectamiento que, hasta entonces, llamaban "declamación poética".

Valora también el metro, el verso bien medido:

Y se convencerá usted que no hay metro, ni hay sentido común en eso de "hartas muellemente" [...] De seis versos, no ha salido, uno solo bien medido (ILS 31, 13).

Rechaza también los excesos debidos al tardorromanticismo:

Sr. R. Cruz Concha (Timón de Carazza). - Tan ciútico (sic) es su pseudónimo como su composición ipor qué diantres Uds. los verseros llorones no tienen otro recurso que "la ingrata me ha olvidado", "jime mi corazón", "soy un trovador" y todas esas vaciedades que se vienen repitiendo desde el $1^{\circ}$ de enero del año 1 (ILS 44, 14).

Destaca la importancia de la relación entre fondo y forma:

Su sueño de poeta, muy bueno, muy bonito, muy barato... pero no publicable, por no tener fondo, superficie ni orillas (ILS 49, 13).

Si Vd. se esmera un poco en la forma escribirá bien $(I 19,13)$.

No sirve; tiene varios versos mal medidos, y las consonantes de la tercera estrofa: batallas, balas metrallas y alas, se parecen demasiado $(I 21,14)$.

Estas apreciaciones sugieren la existencia de un patrón estético común al que se ciñe la valoración crítica de un poema. Algunas veces el contenido afectado, otras veces la forma descuidada, pero en todos los casos se deja entender una cierta perfección de la obra poética como requisito para la publicación.

Hay críticas a la ortografía:

Baldría tener más resignación, dice usted, jvida mía! en sus bersos y creo que á aprender ortografía deberá resignarse usted primero (ILS 56, 13).

A la extensión:

Larga su disertación sobre el amor, recórtela Ud. que bien puede hacerlo, pues hay algunas redundancias que perjudican la originalidad del artículo (ILS 42, 13). 
$\mathrm{O}$ a la originalidad en sospecha:

Para un álbum. Sí, pero los plagios ó robos como el suyo, en ningún álbum deberían admitirlos, aunque sé que es costumbre muy generalizada dedicarle á las niñas versos de otros, sin indicar, por supuesto la paternidad (ILS 61, 12).

También aparecen juicios de género:

Menegilda: Señora: una mujer, por mucho que quiera á un hombre, no se declara ella, sino que espera que se le declaren. Y si no, se muerde y se resigna. [...] Conste, pues, que no se publican sus versos, porque para ser de una mujer son atrevidotes $(I 14,14)$.

Pero la estética que subyace a la crítica de Tío Grulo no sólo se hace ver cuando critica textos que rechaza, sino también cuando invita, con algunos consejos, a mejorar los resultados:

Muy ripioso y rebuscado "El despojo de la estancia" (ILS 26, 12).

El tema no está mal aunque muy explotado, pero está muy malamente tratado en su artículo. Tiene Ud. Condiciones para llegar á escribir algo aceptable. No se desanime (ILS 49, 13).

Daremos su poesía y Ud. dispensará el verso modificado, pero no quisimos tener que rechazarla por su pequeño error (ILS 42, 13).

El mérito de este ejercicio crítico está dado porque, en medio de la gran cantidad de publicaciones rechazadas, en medio de las observaciones de carácter estético, y entre tantos colaboradores cuya creatividad no parece tener mayor valor, comienzan a destacarse algunos nombres. El primero de ellos es Carlos Pezoa Véliz:

Recibidos sus hermosos versos. Se van publicando (ILS 26, 13).

De sus Pensamientos hemos seleccionado algunos. Esperamos no nos olvide Ud., dándole las gracias en nombre de Bórquez por sus inspirados versos (ILS 44, 14).

Recibimos oportunamente su composición "Los ojos de mi amada" que posiblemente va en este número y "La Epopeya del Bozpe", parte II, del inspirado vate (José) Santos Chocano (ILS 49, 13). 
Son numerosos los comentarios dedicados a Pezoa Véliz, al punto que bien podría hipotetizarse que esta revista es su espacio privilegiado de legitimación. Esto queda ratificado por el siguiente comentario:

Encarnación verdadera de poeta trovador, idealista, soñador, sublime, he aquí la exacta definición de la personalidad intelectual de CARLOS PEZOA VELIZ.

Sus versos, de una fluidez admirable y de un lirismo atrayente, encierran toda su alma de poeta y de soñador, transparentándose ora amorosa en dulces y arrobadoras trovas, ora escéptica en cantos llenos de melancolía con crudos arranques de un desnudo realismo que conmueve y fascina á la par. Bohemio consumado, al par que adorador fanático de los grandes maestros, se ha formado un gusto por demás exquisito y delicado para la alta encarnación poética y la selecta literatura, lo que muchas veces lo lleva hasta la intransigencia, aun para consigo mismo, con lo cual ha llegado á atraerse la enemistad de muchos y el casi aislamiento en nuestro campo literario, en donde en fuerza de su exagerada delicadeza artística y su manera de pensar, su nombre no ha logrado conquistarse el puesto á que su talento lo hace merecedor (ILS 35, 4).

Así, Carlos Pezoa Véliz, valorado en su vertiente modernista y descuidado en cuanto a su creación popular -lo cual indica una preferencia estética por parte de los editores-, encuentra en estos semanarios y en el Ateneo espacios de consolidación y de solidificación de su capital específico. Lo mismo ocurre, por citar otro ejemplo, con Mariano Latorre, a quien se le responde en la sección "Correspondencia Particular":

Señor Mariano Latorre Court.- Usted sabe que los pintores japoneses juzgan imprescindible para sus cuadros el riachuelo, la pagoda, el arbolito en punta; pues ustedes, los que no copian la naturaleza, ni se detienen jamás ante una puesta de sol y escriben de memoria con frases de cajón sus crepúsculos, creen que es preciso meter en él: el lago umbrío, las vaporosas sílfides, el brillante Febo, la casta Diana y los yermos campos y la cachimba del mono. Intente usted copiar la naturaleza, agradezca el consejo y si tiene talento ya verá usted qué cambio (ILS 61, 12).

Advertencia que el editor se permite hacer a un escritor en ciernes. Diálogo con el autor, revisión crítica de sus escritos y, en cierta medida, de su poética. Esto ya es un ejercicio crítico que supone avances con respecto a las 
primeras respuestas del Tío Grulo.

Las páginas de Instantáneas ofrecen también una explícita metacrítica, es decir un diálogo del crítico con su oficio, sus funciones, sus desafíos, sus reglas.

Sí que es crítica la situación del crítico que tiene que echarse a cuestas el fardo intelectual de tantos favorecedores de Instantáneas, y que además, pocos ¡ay de mí! valen lo que quisiéramos que valieran para honra y provecho de nuestra tierra, y en particular de esta revista.

Mas esta es mi ingrata tarea y ja la obra!

Pero antes un paréntesis, que aunque redundante considero necesario hacer.

No debe creer el amable colaborador de Instantáneas, que el título de crítica envuelva preconcebida idea de condenar sus trabajos, sino, como lo indica la palabra misma, es el juicio imparcial hecho sobre ellos con arreglo a las reglas del arte y del buen gusto.

$\mathrm{Y}$ aquí me las den todas, subiéndome a tan merecido rango y repartiendo palos, por dulces palos de canela pongo por caso, y aplausos a quien crea en mi bondad de criterio, los merezca...

D. Veritas (ILS 61, 12).

Poco importan los nombres que usan estos anónimos críticos (Teloncillo, Buenafé, Tío Grulo, D. Veritas); lo que importa, precisamente, es el criterio de objetividad que quieren proponer tras el ocultamiento de un nombre biográfico, casi como garantía de distanciamiento y de exención de franquicias debidas a la amistad o a juicios dependientes de cualquier favor o generadores de los mismos. El mismo nombre "Veritas" asigna una pretensión a la actividad crítica como esfuerzo por decir la verdad, esfuerzo no siempre común en la crítica decimonónica, acostumbrada, como dice Alone, a "intercambiar elogios o censuras".

Esta crítica sobre la crítica, correspondiente a la función metacrítica según J. Slawinsky, devela la preconcepción que hacia el 1900 se tenía de los enunciados de crítica literaria y artística, la cual era atendida como una práctica calificadora y marginadora. Si bien la crítica es normativa, según propone Eagleton (1999), bajo el marco de la sociedad del siglo XX, moderna, la función crítica es posible de ser desarrollada por cualquier individuo que despliegue argumentos analíticos y los exponga en alguna de las instancias constitutivas de la esfera pública. El hablante de la cita 
se considera inmerecedor del título de crítico, aunque reconoce que hará uso de su buen criterio para cumplir la labor (Alvarado, 2010: Capítulo IV, s/p).

El hecho de que en estos comentaristas (v.gr.: D. Veritas) ya haya una conciencia crítica, y de que esa actividad deba estar sometida a un cierto reglamento que, bajo el rótulo de "reglas del arte y del buen gusto", responde a parámetros normativos de una época y que, dicho sea de paso, no son sino la apropiación de dos vertientes (una clásica y otra estético-romántica), supone la instalación de actores culturales con un rol mediador específico y, para lo que significa esta revista en particular, fundante, en materia de definiciones, perfiles y configuración del crítico literario.

No obstante, una aclaración debe hacerse y que consiste en que en Instantáneas de luz i sombra son advertibles distintos niveles de actividad crítica y que, en algunos de ellos, se alcanza un nivel de profesionalización que prefigura la estabilización de una función cultural. En este sentido, se pronuncia la profesora Marina Alvarado al relevar la participación de Augusto Thomson en la revista:

Sin embargo, dentro de esta misma revista, quien incursionará con mayor asertividad en el discurso crítico, será Augusto Thomson, el encargado de redactar la columna "Los 21" para Instantáneas de luz i sombra (Alvarado, 2010: Capítulo IV, s/p).

Además, participa escribiendo en algunas columnas que llevaban los títulos de "Arte en el Salón del 1900", “Arte en el Salón de 1901", "Potpurrî". Desde ellas, será posible conocer al futuro Augusto D'Halmar como un conocedor de la escena cultural y literaria no sólo nacional, sino también internacional. Habla de ópera, de zarzuela, de teatro, de escultura, pintura y, sobre todo, de literatura. En su columna "Los 21" hará comentarios de diferentes artistas; entre los chilenos se cuenta a: Virginio Arias, Pedro A. González, Alfredo Valenzuela Puelma, Juan Francisco González, Ernesto Molina, Marcial Plaza Ferrand, Emilio Rodríguez Mendoza, Nicanor Plaza, Diego Dublé Urrutia y Luis Orrego Luco. Quien quiera conocer la escena literaria y artística de principios del siglo XX tiene lectura obligada en las columnas de Thomson. Es imposible construir una historia literaria y cultural chilena sin acudir a los testimonios que dan cuenta de una época en que las letras chilenas se encontraban en ciernes. Thomson, un joven apenas de 1820 años, es el testigo de ese proceso. Y no sólo habla de los que escriben en 
Chile, sino también de aquellos que se leen; es decir, Zola, Tolstoy, Daudet, Maupassant, Dickens, etc.

\section{CONCLUSIONES}

El estudio de las revistas literarias es importante para el conocimiento de los diversos procesos de articulación y desarrollo de todas las instancias vinculadas al campo correspondiente. Ellas informan el día a día y dan cuenta de la minucia práctica que origina un desarrollo posteriormente considerado relevante. En términos concretos, la gran crítica literaria de El Mercurio, que ha dominado la escena cultural chilena por décadas, no se podría entender si, antes de 1906, no se leyeran semanarios o suplementos como Pluma $i$ Lápiz, La ley, La tarde, o los que se han mencionado aquí: Instantáneas, Luz $i$ sombra, Instantáneas de luz i sombra. Entra aquí la consideración bastante clave de que los pequeños semanarios, al estar insertos al interior del campo literario, reflejan, mejor que otros instrumentos de amplia divulgación, la resituación de los distintos actores culturales que contienden por su espacio dentro del campo y, por lo tanto, el capital específico que cada uno de ellos posee.

Queda reafirmado el carácter de la crítica literaria decimonónica como “comentario literario". Andrés Bello, José Victorino Lastarria, Blanco Cuartín, los hermanos Amunátegui, José Toribio Medina, y otros, en realidad no tenían elementos para situarse profesionalmente frente a los textos que analizaban y, además, desde el punto de vista de las categorías de campo, dicha crítica profesional aún no era necesaria, pues no había una real distinción entre creadores y consumidores de literatura ni, por lo tanto, la necesidad de un agente mediador entre ambos.

Se comparte la afirmación de Gonzalo Catalán y de Alone con respecto a que Omer Emeth sería un gran crítico profesional que modificaría las prácticas del oficio. Sin embargo, se discrepa con respecto a si él fue el primero en hacerlo, puesto que la obra crítica de Augusto Thomson en el semanario analizado demuestra varias señales de madurez y solidez.

Instantáneas de luz i sombra, además, cumple con el proceso de transición por otras razones: formaliza la tercerización de juicios sobre las obras que quieren postular a ser publicadas, crea un elenco de evaluadores críticos anónimos de dichas obras, insiste en el carácter de veracidad y objetividad con respecto a la opinión de los críticos en estos casos, crea columnas en donde algunos escritores reconocidos (Thomson, Nadir, Pérez Kallens, entre otros) 
realizan comentarios críticos de mayor vuelo, etc. Gracias a estas columnas será posible conocer la recepción que tenían a inicios de siglo, en Chile, autores como León Tolstoi, Emile Zola, Pierre Loti, Alphonse Daudet, y muchos más que hacía pocas décadas se estaban leyendo en nuestro país.

Finalmente, como síntesis, queda señalar que esta revista es un antecedente que muestra cómo, en un breve período, la crítica literaria adquiere formas cada vez más específicas y que el trayecto que va desde Tío Grulo a Augusto Thomson bien puede considerarse como una preparación para lo que significará el ulterior desarrollo de la crítica literaria chilena.

\section{REFERENCIAS}

Alvarado, Marina. 2010. Revistas Culturales y Literarias chilenas 18941920: Autonomización del Campo Literario y Construcción del Campo de Revistas. Tesis para optar al grado de Doctora en Literatura en la Pontificia Universidad Católica de Valparaíso. Tesis sin publicar.

Catalán, Gonzalo. 1985. "Antecedentes sobre la transformación del campo literario en Chile entre 1890 y 1920”. En Brunner, José Joaquín y Catalán, Gonzalo: Cinco estudios sobre sociedad y cultura. Santiago de Chile: Facultad Latinoamericana de Ciencias Sociales, FLACSO, pp. 70-175.

Díaz Arrieta, Hernán (Alone). 1931. Panorama de la literatura chilena durante el siglo $X X$. Santiago: Nascimento, $181 \mathrm{p}$.

Dyson, John P. 1965. La evolución de la crítica literaria en Chile. Santiago de Chile: Editorial Universitaria.

Fernández Fraile, Maximino. 2003. La Crítica Literaria en Chile. $1^{\text {a }}$ edición. Santiago de Chile: Editorial Don Bosco, 353 p.

Instantáneas de Luz i sombra: semanario festivo, literario, artístico y de actualidades. 1900-1901. Santiago: Imprenta Barcelona. Abreviado como ILS.

Lastra, Pedro. 2000. Leido y anotado: letras chilenas e hispanoamericanas: imágenes / encuentros. $1^{a}$ edición. Santiago de Chile: LOM, 168 p.

Silva Castro, Raúl. 1969. La Literatura critica de Chile: antología con estudio preliminar. Santiago: Andrés Bello, 530 p.

Vaïsse, Emilio. 1909. La vida literaria en Chile: primera serie 1908-1909. Santiago de Chile: El Mercurio, 422 p. 